\title{
Recruitment of Brd3 and Brd4 to acetylated chromatin is essential for proinflammatory cytokine-induced matrix-degrading enzyme expression
}

\author{
Jin Dai ${ }^{1 \dagger}$, Sheng Zhou ${ }^{1 \dagger}$, Qiting Ge ${ }^{2}$, Jinzhong Qin ${ }^{2}$, Jianxin $\mathrm{Li}^{3}$, Huangxian Ju${ }^{3}, \mathrm{Yi} \mathrm{CaO}^{4}$, Minghao Zheng ${ }^{5}$,
} Chaojun Li ${ }^{6}$, Xiang Gao ${ }^{2}$, Huajian Teng ${ }^{1,2^{*}}$ and Qing Jiang ${ }^{1,2,3^{*}}$ (D)

\begin{abstract}
Background: Proinflammatory cytokines, which can upregulate the expression of matrix-degrading enzymes in chondrocytes, play important roles in the development of osteoarthritis. BET family proteins, acting as the "readers" of acetylated modifications on histones, have been linked to transcriptional regulation. And a BET protein inhibitor, I-BET151, has been shown to inhibit the induction of matrix-degrading enzymes by proinflammatory cytokines in chondrocytes. Our objective is to clarify the role and mechanism of BET proteins on matrix-degrading enzyme gene expression by using a human chondrosarcoma cell line (SW1353).

Methods: We pretreated SW1353 cells with I-BET151 prior to treatment with IL-1 $\beta$ or TNF-a and then checked the expression of four matrix-degrading enzyme genes (MMP1, MMP3, MMP13, and ADAMTS4). We performed knockdown of BET protein family members (BRD2, BRD3, and BRD4) with corresponding siRNAs in SW1353 cells prior to treatment with IL-1 $\beta$ or TNF- $\alpha$ and checked the expression of the matrix-degrading enzyme genes. We evaluated Brd-mediated transcriptional regulation on the matrix-degrading enzyme genes by ChIP assay.

Results: We confirmed that I-BET151 could suppress the IL-1 $\beta$ - or TNF-a-induced expression of MMP1, MMP3, MMP13, and ADAMTS4 in SW1353 cells. Brd3 and Brd4 were required for the IL-1 $\beta$ - or TNF-a-induced expression of matrixdegrading enzyme genes in SW1353 cells. We revealed that inducible acetylation of H4k5/8/12 and the recruitment of $\mathrm{Brd} 3, \mathrm{Brd} 4$, and $\mathrm{p}-\mathrm{TEFb}$ to chromatin were involved in IL-1 $\beta$ - or TNF- $\alpha$-induced transcription.

Conclusions: Our findings suggested that Brd3 and Brd4 were essential for the IL-1 $\beta$ - or TNF-a-induced transcription of matrix-degrading enzyme genes, and recruitment of $\mathrm{Brd} 3$ and $\mathrm{Brd} 4$ to chromatin of these genes played the main role in this process.
\end{abstract}

Keywords: Matrix-degrading enzymes, Brd, Transcription, Acetylation

\section{Background}

Osteoarthritis (OA) is a common multifactorial disorder of the joints and is mainly characterized by the progressive degeneration of articular cartilage. Matrix-degrading enzymes including matrix metalloproteinase (MMP) 1, MMP3, MMP13, a disintegrin and metalloproteinase with

\footnotetext{
*Correspondence: tenghj@hotmail.com; qingj@nju.edu.cn

${ }^{\dagger}$ Jin Dai and Sheng Zhou contributed equally to this work.

${ }^{1}$ The Center of Diagnosis and Treatment for Joint Disease, Drum Tower Hospital, Medical School, Nanjing University, Zhongshan Road 321, Nanjing 210008, Jiangsu, People's Republic of China

Full list of author information is available at the end of the article
}

thrombospondin motifs (ADAMTS) 4, and ADAMTS5 played important roles in cartilage degradation in OA [1]. Among these matrix-degrading enzymes, MMP1, MMP3, MMP13, and ADAMTS4 can be upregulated by proinflammatory cytokines, such as interleukin (IL)- $1 \beta$ and tumor necrosis factor (TNF)- $\alpha$. Signal transduction pathways, such as the nuclear factor- $\mathrm{kB}(\mathrm{NF}-\mathrm{kB})$, Jun $\mathrm{N}$-terminal kinase (JNK), and mitogen-activated protein kinase (MAPK) pathways, were involved in the process of activating matrix-degrading enzymes by proinflammatory cytokines [1-3]. 
Recently, a small molecular inhibitor which could block bromodomain and extraterminal domain (BET) proteins from recognizing acetylated histones (I-BET151) was generated [4], and we found I-BET151 could abrogate the induction of MMP1, MMP3, MMP13, and ADAMTS4 by IL-1 $\beta$ or TNF- $\alpha$ in chondrocytes [5]. In mammals, the BET family of proteins consists of ubiquitously expressed bromodomain-containing protein (Brd)2, Brd3, Brd4, and testes/oocyte-specific Brdt. All members of BET proteins contain two conserved tandem bromodomains capable of recognizing acetylated histones, which are involved in transcription regulation through the recruitment of transcriptional regulatory complexes to acetylated chromatin $[6,7]$. There were studies which reported that IL- $1 \beta$ and TNF- $\alpha$ could increase the acetylation of histones within the promoter regions of different genes [8-10]. We suspected that enhanced recruitment of BET proteins to acetylated histones at the promoter regions was responsible for the induction of these matrix-degrading enzymes by IL- $1 \beta$ or TNF- $\alpha$.

In the present study, we examined the role and mechanisms of BET proteins in the transcriptional regulation of matrix-degrading enzyme genes. We demonstrated that recruitment of BET proteins to acetylated chromatin is a crucial step for the induction of these matrix-degrading enzymes by IL- $1 \beta$ or TNF- $\alpha$, and only Brd3 and Brd 4 had a positive effect in this process.

\section{Methods}

\section{Cell culture and treatment}

Human chondrosarcoma cells (SW1353) were obtained from ATCC and cultured in Dulbecco's Modified Eagle's Medium (Invitrogen) supplemented with $10 \%$ fetal bovine serum (Invitrogen), $2 \mathrm{mM}$ glutamine, 100 units $/ \mathrm{ml}$ penicillin, and $100 \mathrm{~g} / \mathrm{ml}$ streptomycin at $37{ }^{\circ} \mathrm{C}$ in an atmosphere of 5\% $\mathrm{CO}_{2}$. Although SW1353 cell line has limitations for chondrocyte research, this cell line has been shown to be a suitable cell model for examining the regulation of many catabolic genes in chondrocytes [11], and high transfection efficiency can be obtained in this cell line. Human samples were obtained with informed consent from the donors. The study was approved by the ethical committee of Drum Tower Hospital, Medical School, Nanjing University. Fresh cartilage samples (from a patient who underwent knee replacement surgery at Drum Tower Hospital, Medical School, Nanjing University) were chopped from the lateral condyle of the operated knee. The patient was male and 65 years of age. He suffered with OA and had no history of immunological diseases. Primary human articular chondrocytes were cultured as previously described [12].

For the cellular assays, the cells were grown to approximately $80 \%$ confluence and then starved (in medium containing $0.1 \% \mathrm{FBS})$ for $12 \mathrm{~h}$. I-BET151 $(1 \mu \mathrm{M}$; TOCRIS, $\mathrm{R} \& \mathrm{D}$ Systems) or vehicle was added $1 \mathrm{~h}$ prior to treatment with IL-1 $\beta$ (10 ng/ml; R\&D Systems) or TNF- $\alpha$ (10 ng/ml;
R\&D Systems). Dimethyl sulfoxide (DMSO) was used as the vehicle for I-BET151, IL-1 $\beta$, and TNF- $\alpha$.

\section{Gene expression analysis}

The total RNA from primary human chondrocytes or SW1353 cells was isolated using TRIzol Reagent (Ambion, Invitrogen). First-strand cDNA was prepared by reverse transcription using the PrimeScript RT Reagent Kit according to the manufacturer's manual (TaKaRa). Real-time PCR was performed in an ABI StepOnePlus instrument (Applied Biosystems) using SYBR Green PCR Master Mix (Thermo Scientific). Briefly, all real-time PCR was performed in a reaction volume of $20 \mu \mathrm{l}$, using 96-well optical grade PCR plates (Invitrogen). The reactions were conducted at $50{ }^{\circ} \mathrm{C}$ for $2 \mathrm{~min}$, then at $95^{\circ} \mathrm{C}$ for $10 \mathrm{~min}$, and followed by $40 \mathrm{cy}$ cles of $95^{\circ} \mathrm{C}$ for $15 \mathrm{~s}$ and at $60^{\circ} \mathrm{C}$ for $60 \mathrm{~s}$. The primers used in this study are listed in Additional file 1: Table S1. Each sample for real-time PCR was evaluated by three tests.

\section{RNA interference}

Predesigned siRNAs specific to human $B R D 2, B R D 3$, and $B R D 4$, as well as scrambled siRNA, were obtained from Santa Cruz Biotech (Santa Cruz Biotechnology). SW1353 cells were grown to approximately $70 \%$ confluency and were then treated with siRNA using Lipofectamine 2000 for $48 \mathrm{~h}$ prior to treatment with IL-1 $\beta$ or TNF- $\alpha$.

\section{Western blot assay}

Western blot assays were carried out as previously described [13]. The proteins were resolved on $8-12 \%$ SDS-polyacrylamide gels, as required, and transferred to BioTrace NT membranes (Pall, Life Sciences). The membranes were probed with the appropriate primary antibodies, and the proteins were detected using peroxidase-conjugated antibodies and visualized by ECL (Pierce, Thermo). The primary antibodies used were rabbit polyclonal anti-Brd4 (1:1000; Cell Signaling Technology), rabbit monoclonal anti-Brd2 (1:1000; Abcam), mouse monoclonal anti-Brd3 (1:500; Abcam), and anti-glyceraldehyde-3-phosphate dehydrogenase (GAPDH) (1:1000; Santa Cruz Biotechnology, INC).

\section{ChIP assay}

ChIP assays were carried out using a commercially available kit according to the manufacturer's instructions (Upstate, Millipore). Briefly, human chondrocytes were fixed, harvested, and sonicated. The sonicated supernatants were diluted with ChIP dilution buffer and precleared with protein $G$ agarose. After removing $1 \%$ supernatants as input, the supernatants were incubated with an antibody against Brd3 (Abcam), Brd4 (Abcam), cyclin-dependent kinase 9 (Cdk9) (Santa Cruz Biotechnology, INC), Ser2-phosphorylated RNA polymerase II C-terminal domain (S2P Pol II) (Abcam), H4K5Ac 
(Millipore), H4K8Ac (Millipore), H4K12Ac (Millipore), and nonspecific IgG (Upstate, Millipore) antibodies overnight at $4{ }^{\circ} \mathrm{C}$ with rotation. The protein/DNA complexes and the input were eluted, reversed, and purified. The quantitative analysis of targeted promoter regions was determined by real-time PCR using specific primers (Additional file 1: Table S1), and the results were normalized to the level of input by using the same primers. Each sample for real-time PCR was evaluated by three tests.

\section{Statistical analysis}

All data were expressed as the means SE and represent at least two independent experiments. Statistical comparisons were made using Student's $t$ test. $P<0.05$ was considered statistically significant.

\section{Results}

Brd3 and Brd4 are required for expression of IL-1 $\beta$ - or TNF-a-induced matrix-degrading enzymes

We first verified that transcriptional expression of $M M P 1$, $M M P 3, M M P 13$, and ADAMTS4 was upregulated by IL-1 $\beta$ or TNF- $\alpha$ and repressed by I-BET151 in a human chondrosarcoma cell line (SW1353) (Fig. 1). We then examined the expression of BET proteins by using Western blot assay (Additional file 2: Figure S1) and found the patterns of Brd2, Brd3, and Brd4 protein expression were similar in SW1353 cells and primary human chondrocytes (Fig. 2a). Finally, we performed a knockdown of $B R D 2, B R D 3$, and $B R D 4$ with corresponding siRNAs in SW1353 cells and confirmed the reduction of Brd2, Brd3, and Brd4 by using Western blot assay (Fig. 2b-d). The analysis of the transcription levels revealed an apparent reduction of inducible transcription of $M M P 1, M M P 3, M M P 13$, and ADAMTS4 in the BRD3 and
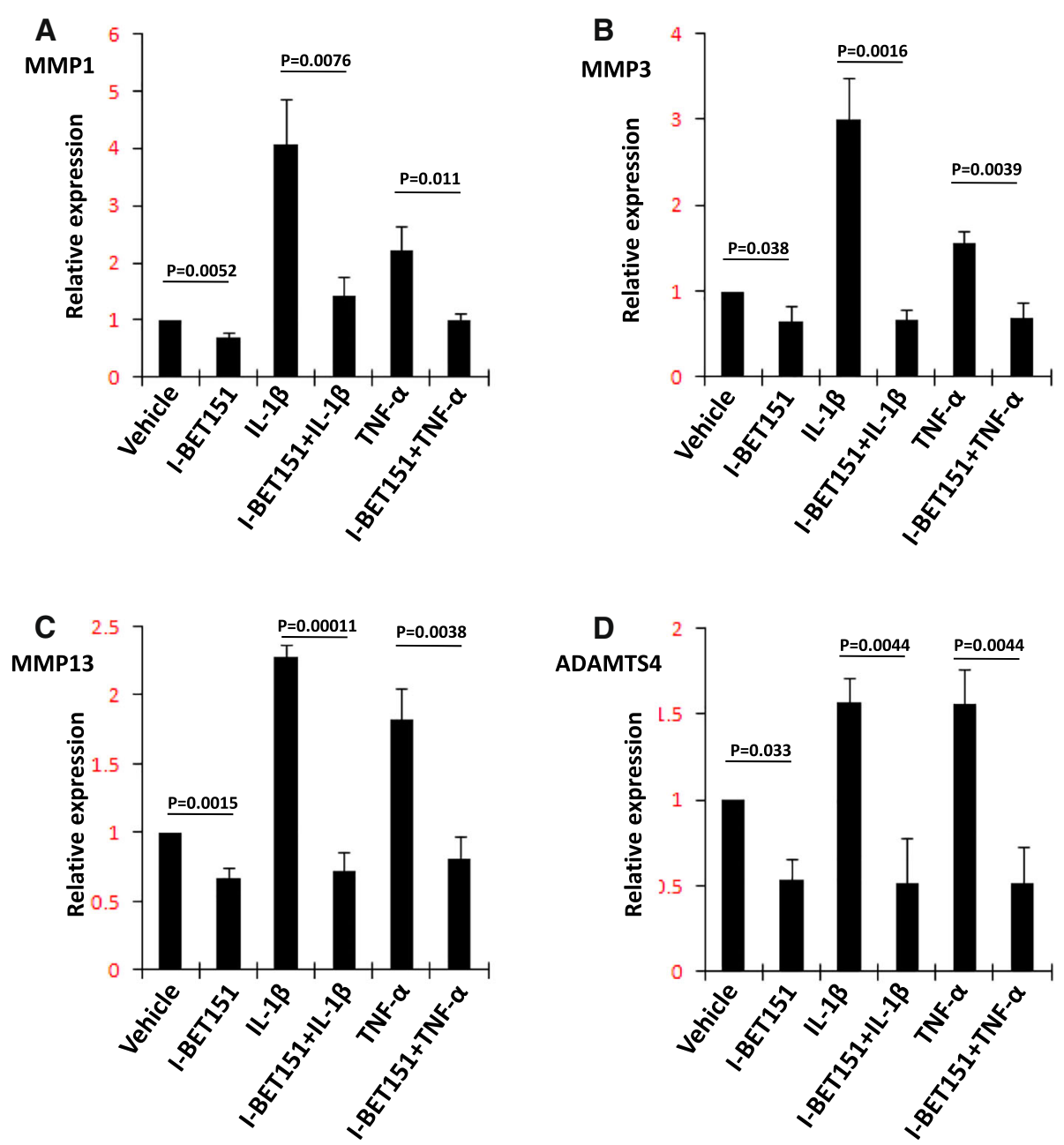

Fig. 1 The effect of I-BET151 on regulation of matrix-degrading enzyme gene transcription in SW1353 cells. a-d The transcriptional expression (RT-PCR) of MMP1, MMP3, MMP13, and ADAMTS4 genes in SW1353 cells, respectively, after the cells were pretreated with or without I-BET151 $(1 \mu \mathrm{M})$ followed by addition of vehicle, IL-1 $\beta(10 \mathrm{ng} / \mathrm{ml})$ or TNF-a $(10 \mathrm{ng} / \mathrm{ml})$ for $6 \mathrm{~h}$. Relative fold-change values were calculated in comparison with vehicle control that was set to $1(n=3)$ 


\section{A}

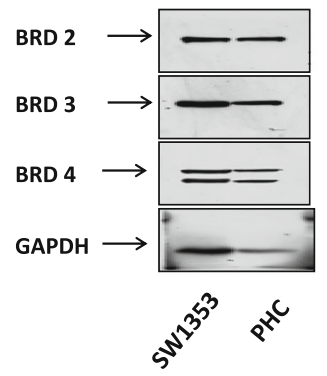

C



B

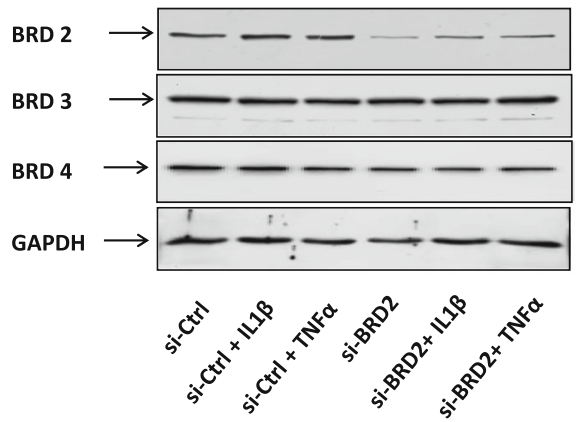

D

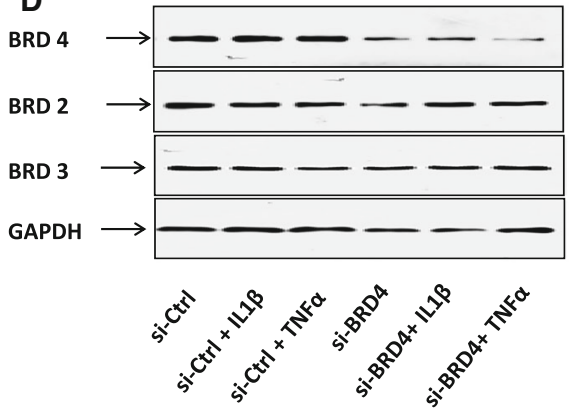

Fig. 2 The protein expression of the BET family members and the effect of siRNAs in SW1353 cells. a The protein expression of Brd2, Brd3, and Brd4 was confirmed by Western blot assay in SW1353 cells and primary human chondrocytes, respectively. PHC, primary human chondrocyte. bd SW1353 cells were treated with scrambled siRNA or specific siRNA targeting BRD2, BRD3, and BRD4 for $48 \mathrm{~h}$ and then treated with or without IL-1 $\beta(10 \mathrm{ng} / \mathrm{ml})$ or TNF-a $(10 \mathrm{ng} / \mathrm{ml})$ for $6 \mathrm{~h}$. The presence of Brd2, Brd3, and Brd4 proteins in SW1353 cells was confirmed by Western blot assay

BRD4 knockdown cells following stimulation. The reduction of IL-1 $\beta$-induced $M M P 3$ transcription, TNF- $\alpha$-induced $M M P 3$ transcription, and TNF- $\alpha$-induced ADAMTS4 by $B R D 3$ knockdown and the reduction of $\mathrm{IL}-1 \beta$-induced $M M P 3$ transcription by BRD4 knockdown did not reach the criteria of significance, but all the comparisons showed the same trends. BRD2 knockdown resulted in increased levels of basal and inducible transcription of $M M P 1, M M P 3$, and $M M P 13$ but not of ADAMTS4 (Fig. 3a-d).

\section{IL-1 $\beta$ - or TNF- $\alpha$-induced recruitment of Brd3 and Brd4 to chromatin}

We evaluated the recruitment of Brd3 and Brd4 to the promoter regions of $M M P 1, M M P 3, M M P 13$, and ADAMTS4 by ChIP assay and found enhanced recruitment of Brd3 and Brd4 to the promoter regions of all the four genes after treatment of IL- $1 \beta$ or TNF- $\alpha$. The average fold changes were all larger than 2.0, except the recruitment of Brd3 to MMP3 after the treatment of IL-1 $\beta$ and the recruitment of Brd3 to MMP3 and Brd4 to ADAMTS4 after the treatment of TNF- $\alpha$. I-BET151 abrogated the enhanced recruitment of $\operatorname{Brd} 3$ and $\operatorname{Brd} 4$ to the promoter regions induced by IL- $1 \beta$ or TNF- $\alpha$, and significance was found in the reduction of IL-1 $\beta$-induced recruitment of Brd3 to MMP13 and TNF- $\alpha$-induced recruitment of Brd3 to $M M P 1, \operatorname{Brd} 3$ to $M M P 13, \operatorname{Brd4}$ to $M M P 1$, and Brd4 to
MMP13. But I-BET151 did not show consistent effects on the basal recruitment of $\mathrm{Brd} 3$ and $\mathrm{Brd} 4$ to the promoter regions of these genes (Fig. $4 \mathrm{a}-\mathrm{h}$ ).

We also evaluated the recruitment of CDK9 and the Ser2 phosphorylation of RNAP II CTD at the promoter regions of these genes. In parallel with the recruitment of Brd3 and Brd4 to the promoter regions of these genes, we found that IL- $1 \beta$ or TNF- $\alpha$ stimulation led to increased recruitment of CDK9 and Ser2 phosphorylation of RNAP II CTD in all the four genes (Fig. 5a-h). The average fold changes were all larger than 2.0. I-BET151 abrogated both the recruitment of CDK9 and the Ser2 phosphorylation of RNAP II CTD induced by IL- $1 \beta$ or TNF- $\alpha$, and significance was found in the reduction of TNF- $\alpha$-induced recruitment of CDK9 to MMP1, IL-1 $\beta$-induced recruitment of CDK9 to ADAMTS4, TNF- $\alpha$-induced Ser2 phosphorylation of RNAP II CTD in $M M P 3$, and IL-1 $\beta$-induced Ser2 phosphorylation of RNAP II CTD in MMP1, MMP3, and MMP13. I-BET151 did not show consistent effects on the basal recruitment of CDK9 to the promoter regions or basal Ser2 phosphorylation of RNAP II CTD in these genes (Fig. 5a-h).

\section{IL-1 $\beta$ - or TNF- $\alpha$-induced modifications of acetylated histone at promoter regions}

We checked whether IL- $1 \beta$ or TNF- $\alpha$ stimulation could affect these histone modifications at the promoter regions 


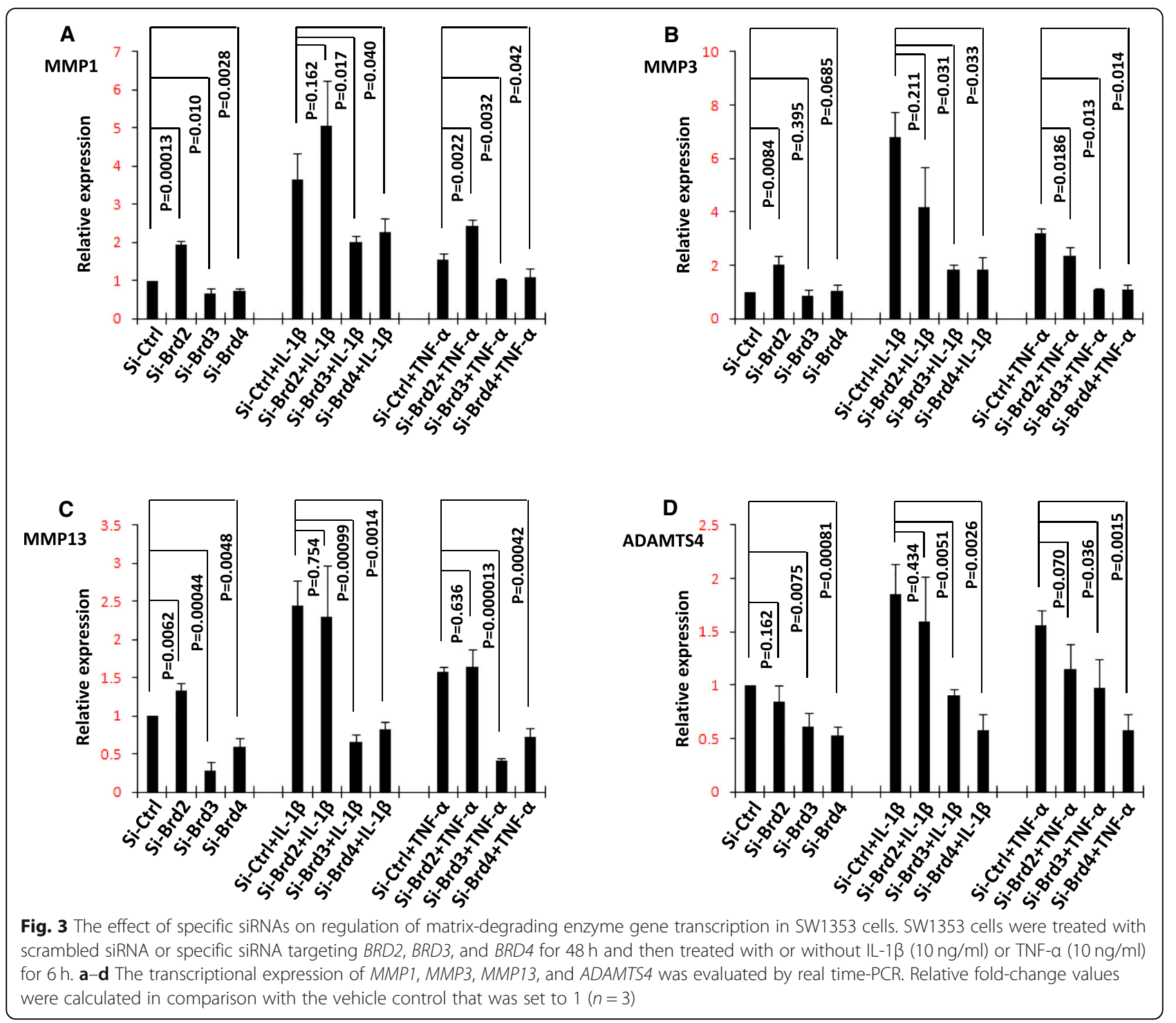

of these inducible genes in chondrocytes, and we found increased levels of these modifications present at $M M P 1$, $M M P 3, M M P 13$, and ADAMTS4 in promoter regions. Significance was found in the IL-1 $\beta$-induced H4K5Ac at $M M P 1, \mathrm{H} 4 \mathrm{~K} 5 \mathrm{Ac}$ at MMP3, and H4K12Ac at ADAMTS4 and in the TNF- $\alpha$-induced H4K8Ac at MMP1 and $\mathrm{H} 4 \mathrm{~K} 12 \mathrm{Ac}$ at $M M P 3$. The average fold changes of all the induced modifications present at $M M P 1, M M P 3$, and $M M P 13$ were larger than 1.5 , except TNF- $\alpha$ induced H4K5Ac at MMP3. The average fold changes of all the induced modifications present at ADAMTS4 were between 1 and 1.5(Fig. 6a-c).

\section{Discussion}

In our study, we found that the depletion of Brd4 and Brd3 by siRNA-mediated knockdown resulted in the reduction of IL1 $\beta$ - or TNF $\alpha$-induced transcription of
MMP1, MMP3, MMP13, and ADAMTS4 in chondrocytes, but depletion of Brd2 did not show such effects. It suggested that both Brd3 and Brd4 were responsible for the IL1 $\beta$ - or TNF $\alpha$-induced transcription of these genes in chondrocytes.

Increasing amounts of evidence suggest that the recruitment of BET proteins to chromatin is closely associated with their roles in transcriptional regulation $[6,7]$. Here, we found enhanced recruitment of Brd3 and Brd4 to the promoter regions of $M M P 1, M M P 3, M M P 13$, and ADAMTS4 after the treatment of IL- $1 \beta$ or TNF- $\alpha$ in chondrocytes, and the recruitment could be alleviated by I-BET151. The recruitment of Brd3 and Brd4 is consistent with the expression of these four genes after the treatment of IL-1 $\beta$ or TNF- $\alpha$ in chondrocytes. So, we suspected that the recruitment of Brd3 and Brd4 to the promoter regions was essential for the induction of 

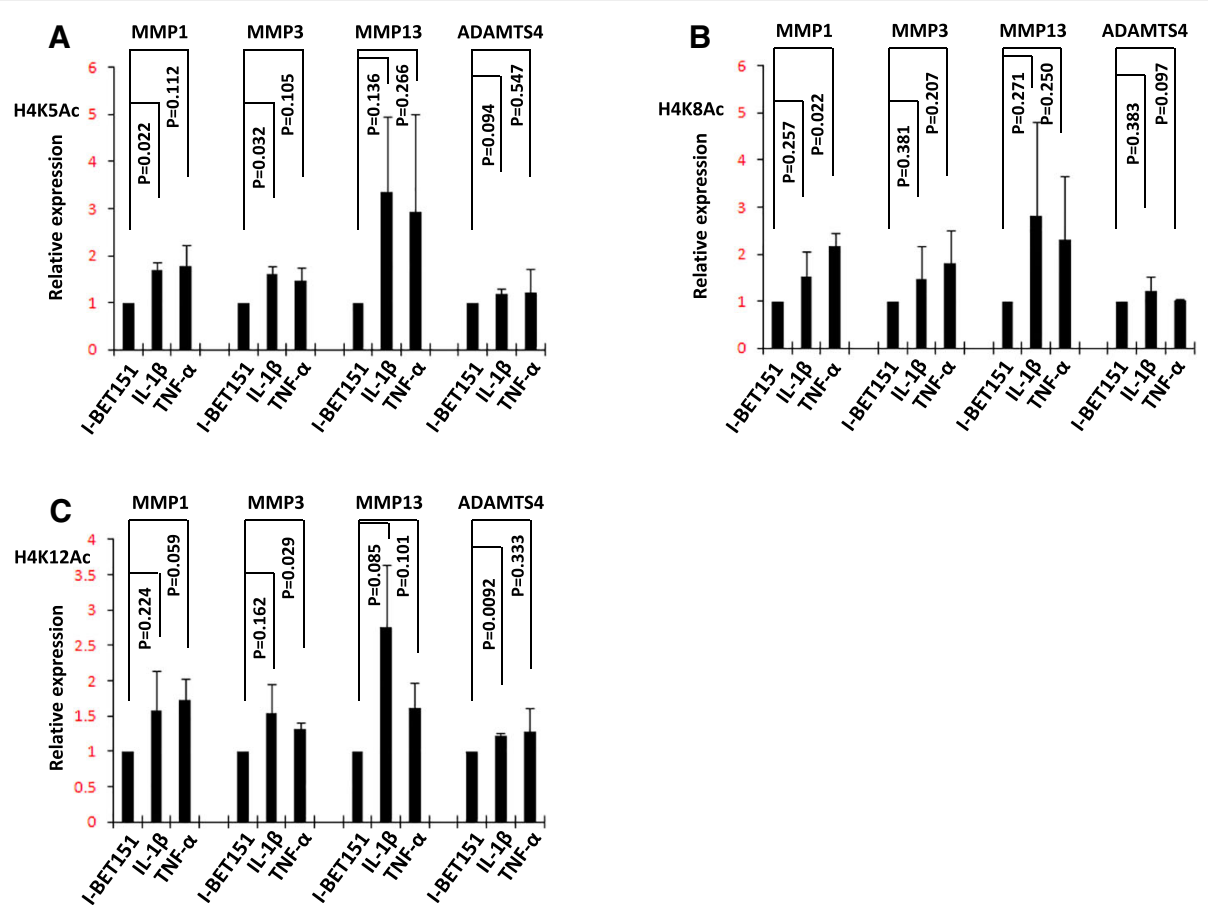

Fig. 4 The recruitment of Brd3 and Brd4 to the chromatin responsible for IL-1 $\beta$ - or TNF- - -induced transcription. a-d Human chondrocytes were pretreated with or without I-BET151 $(1 \mu \mathrm{M})$, followed by addition of vehicle, IL-1 $\beta(10 \mathrm{ng} / \mathrm{ml})$ or TNF-a $(10 \mathrm{ng} / \mathrm{ml})$ for $6 \mathrm{~h}$, and ChIP assays were

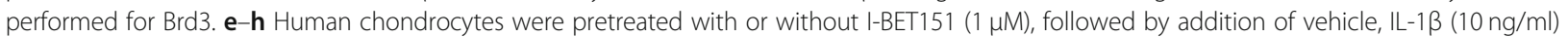
or TNF-a $(10 \mathrm{ng} / \mathrm{ml})$ for $6 \mathrm{~h}$, and ChIP assays were performed for Brd4. The quantitative analysis of targeted promoter regions was determined by real-time PCR using specific primers for MMP1, MMP3, MMP13, and ADAMTS4. Relative fold-change values were calculated in comparison with the vehicle control that was set to $1(n=2)$

MMP1, MMP3, MMP13, and ADAMTS4 by IL-1 $\beta$ or TNF- $\alpha$ in chondrocytes.

There have been studies which reported that Brd4 could directly interact with positive transcription elongation factor $\mathrm{b}$ (p-TEFb) $[14,15]$. p-TEFb is composed of the CDK9 and cyclin T1 (CycT1) subunits, and CDK9 could phosphorylate Ser2 of the RNA polymerase II C-terminal domain (RNAP II CTD), leading to the release of paused RNAP II during transcriptional elongation [16]. In parallel with enhanced recruitment of Brd3 and Brd4 to the promoter regions of $M M P 1$, $M M P 3, M M P 13$, and ADAMTS4, we found enhanced recruitment of CDK9 and Ser2 phosphorylation of RNAP II CTD at the promoter regions of all the four genes after the treatment of IL- $1 \beta$ or TNF- $\alpha$ in chondrocytes. When we inhibited the combination of BET proteins and acetylated histones by using I-BET151 in chondrocytes, we found that the IL-1 $\beta$ - or TNF- $\alpha$-induced recruitment of Brd3, Brd4, and CDK9 to the promoter regions and Ser2 phosphorylation of RNAP II CTD at the promoter regions of these genes were abrogated synchronously. Our results conformed to the previous reports that Brd4 could directly interact with $\mathrm{p}$-TEFb and p-TEFb phosphorylated Ser2 of the RNAP II CTD. The results suggested that the recruitment of Brd4, Brd3, and pTEFb to the chromatin and subsequent Ser2 phosphorylation of RNAP II CTD are correlated with the IL-1 $\beta$ - or TNF- $\alpha$-induced transcriptional expression of $M M P 1, M M P 3, M M P 13$, and ADAMTS4 genes in human chondrocytes.

BET proteins directly interact with several different modifications of acetylated histones, including $\mathrm{H} 4$ acetylated at Lys5 (H4K5Ac), H4 acetylated at Lys8 (H4K8Ac), and H4 acetylated at Lys12 (H4K12Ac) [1719]. Here, we also found increased levels of acetylated H4K5/8/12 and enhanced recruitment of Brd3 and Brd4 to the promoter regions of $M M P 1, M M P 3, M M P 13$, and ADAMTS4 after the treatment of IL- $1 \beta$ or TNF- $\alpha$ in chondrocytes. Our results suggested that increased levels of acetylated $\mathrm{H} 4 \mathrm{~K} 5 / 8 / 12$ at promoter regions might be responsible for the enhanced recruitment of $\mathrm{Brd} 3$ and $\mathrm{Brd} 4$ to the promoter regions of these genes after the treatment of IL-1 $\beta$ or TNF- $\alpha$ in chondrocytes.

Acetylation of histones was reported to be involved in the induction of matrix-degrading enzyme genes by proinflammatory cytokines. There were several reports which suggested NF-kB, JNK, and MAPK pathways were involved in the acetylation of histones [10, 20, 21], and NF- $\mathrm{kB}$, JNK, and MAPK pathways were well known to be involved in the process of activating matrix-degrading 


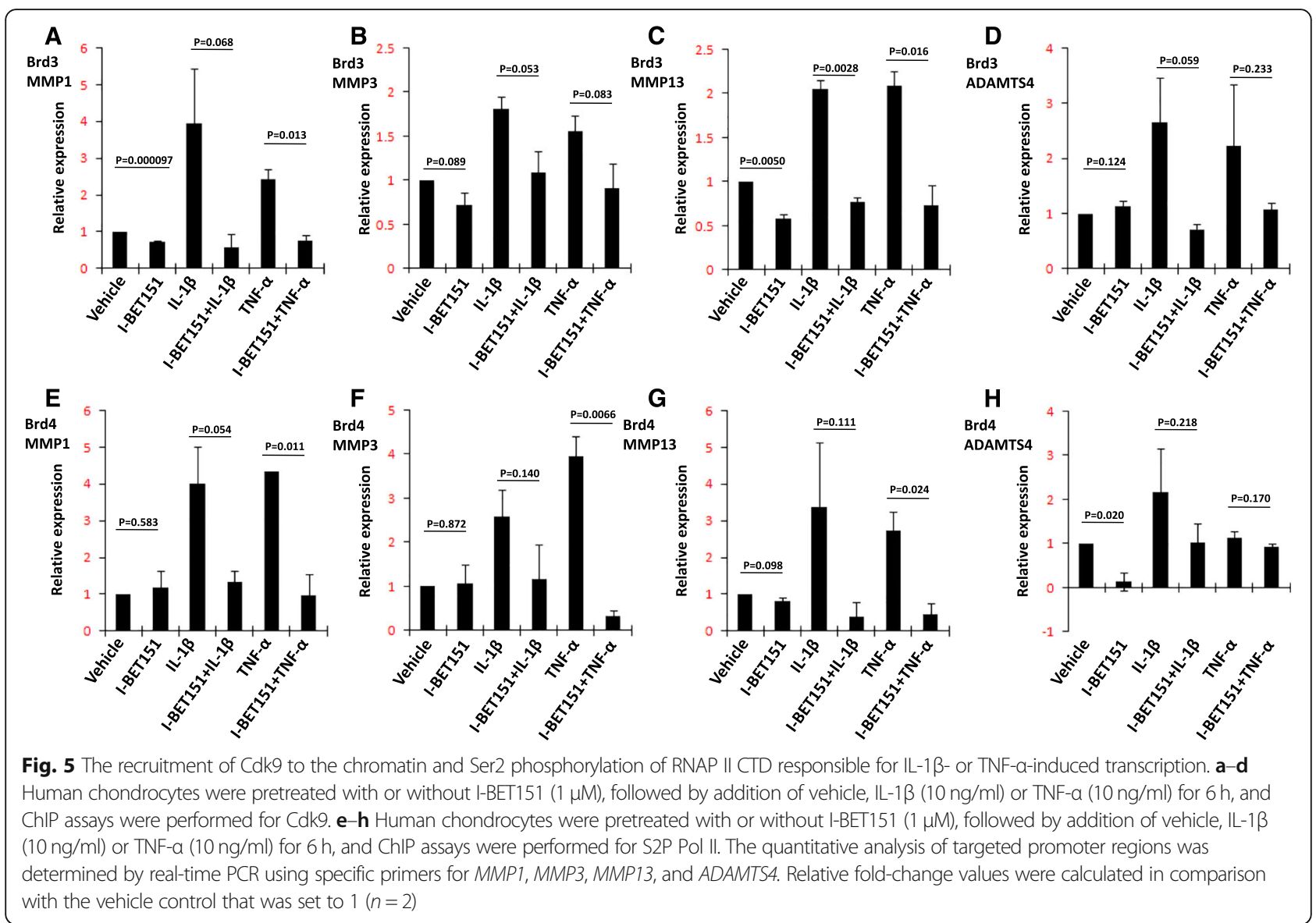

enzymes by proinflammatory cytokines [1-3]. TNF could increase the acetylation of histone $\mathrm{H} 3$ at the promoter region of another matrix-degrading enzyme, MMP9, and then promote the transcription of MMP9 [10]. But the inhibitor of HDAC, which could retain the acetylation of histones, was well known to repress the induction of MMPs by proinflammatory cytokines [2224]. The effect of the inhibitor of HDAC might be mediated by acetylation of $\alpha$-tubulin, acetylation of NF- $\kappa B$ $\mathrm{p} 65$, and so on $[23,25]$. So, the effect of the acetylation of histones on the induction of matrix-degrading genes by proinflammatory cytokines is still debatable and complicated, and much of the mechanism remains unknown. Our results suggested that increased levels of acetylated $\mathrm{H} 4 \mathrm{~K} 5 / 8 / 12$ at promoter regions of $M M P 1, M M P 3$, $M M P 13$, and ADAMTS4 genes were associated with enhanced transcriptional expression of these genes in chondrocytes after the treatment of IL- $1 \beta$ or TNF- $\alpha$.

We found the BET proteins, which acted as "readers" of the histone acetylation, were involved in this process. Brd3 and Brd4 were both shown to be essential for the inducible transcription of $M M P 1, M M P 3, M M P 13$, and ADAMTS4 in chondrocytes after the treatment of IL-1 $\beta$ or TNF- $\alpha$. Enhanced recruitment of Brd3 and Brd4 to the promoter regions could lead to the enhanced recruitment of p-TEFb to the promoter regions and the subsequent Ser2 phosphorylation of RNAP II CTD, and then lead to the enhanced transcription of these genes after the treatment of IL-1 $\beta$ or TNF- $\alpha$.

Some limitations should be noted in our study. First, because of the limited test times and variety of ChIP assay result, significance was not detected in several comparisons although the trend and fold change were apparent. And we did not perform an electrophoresis assay to get a qualitative ChIP data, which could show visual differences among different treatment groups. Second, we only checked the transcriptional level of the target genes, and a protein level presentation could make the effect of treatments more convincing in this study. And we only used one effective siRNA for each Brd protein to perform the knockdown treatment, although the knockdown effects have been confirmed by Western assay. Third, we suggested the association between increased levels of acetylated H4K5/8/12 and the enhanced recruitment of Brd3 and Brd4 to the promoter regions depending on the previous reports that BET proteins could directly interact with several different modifications of acetylated histones, including acetylated histones 

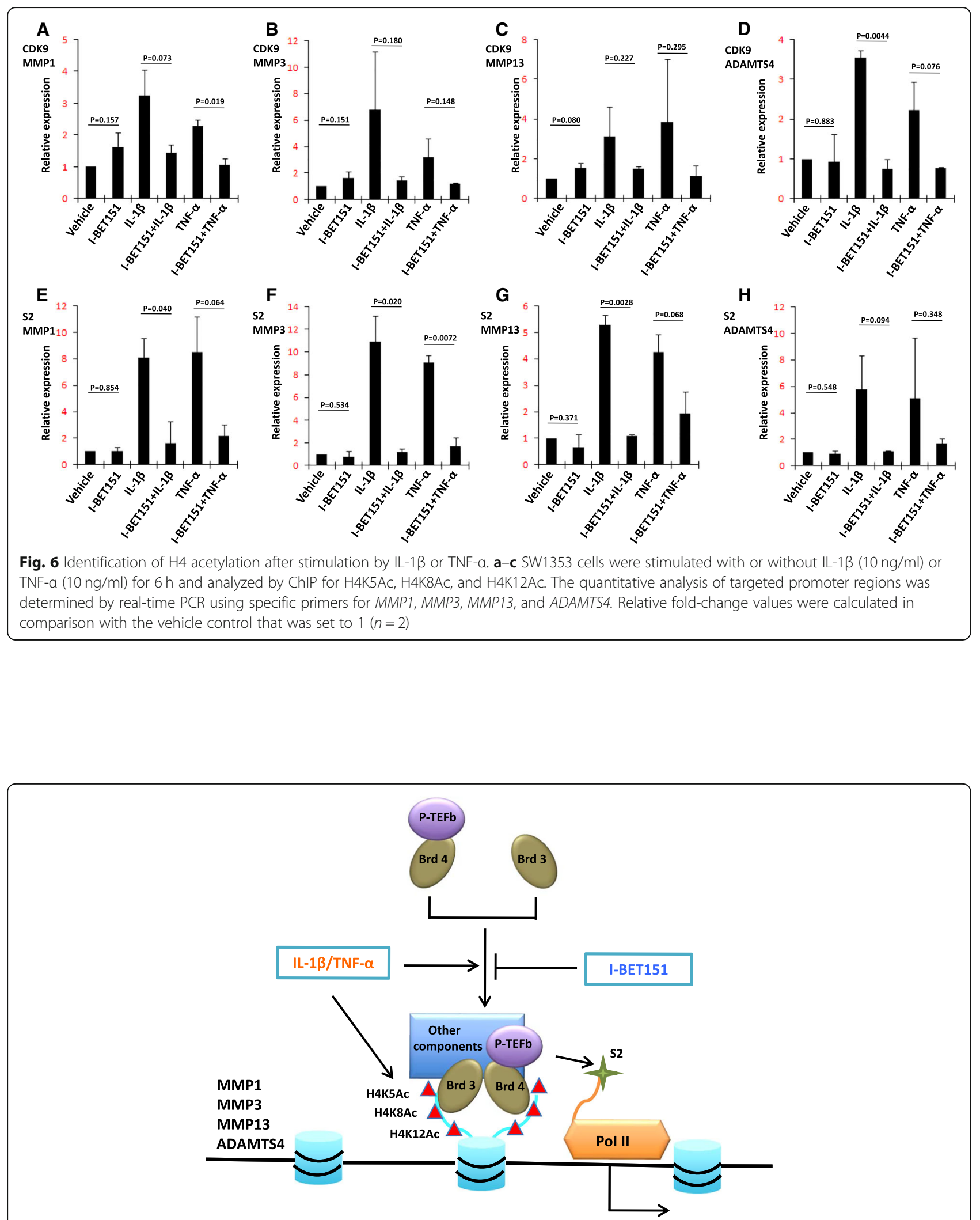

Fig. 7 Schematic mechanism for Brd-mediated transcriptional regulation of MMP1, MMP3, MMP13, and ADAMTS4 genes in human chondrycytes. This model proposes that IL-1 $\beta$ or TNF-a stimulation leads to the H4K5/8/12Ac at TSS regions and the recruitment of Brd3, Brd4, and p-TEFb to these regions. Subsequently, Ser2 RNAP II CTD is phosphorylated by p-TEFb. The recruitment of Brd3 and Brd4 is blocked by I-BET-151 
H4 [17-19]. But we did not specifically inhibit the acetylation of histones $\mathrm{H} 4$ to evaluate the necessity of acetylated histones $\mathrm{H} 4$ in this process. Besides, we cannot deny the enhanced acetylation of other histones at the promoter region or any other mechanisms increasing the affinity of BET proteins to the promoter region as the reason for the enhanced recruitment of $\operatorname{Brd} 3$ and Brd4 to the promoter regions of these genes in chondrocytes. The exact binding sites of Brd3 and Brd4 were still unknown in our study, and further structural analysis was needed to understand the exact mechanisms.

\section{Conclusions}

In summary, the results of the present study showed Brd3 and Brd4 were both characterized to be required for IL-1 $\beta$ or TNF- $\alpha$-induced transcription of matrix-degrading enzyme genes in chondrocytes. Through recognizing acetylated histone, Brd4 might cooperate with Brd3 to recruit $\mathrm{p}-\mathrm{TEFb}$ to the chromatin and then phosphorylate the Ser2 of RNAP II CTD in these induced genes during transcriptional activation (Fig. 7).

\section{Additional files} Additional file 1: Table S1. The list of primers used in this study (DOC
$36 \mathrm{~kb})$

Additional file 2: Figure S1. The protein expression of the BET family members and the effect of siRNAs in SW1353 cells (A) The relative densitometry of Fig. $2 \mathrm{a}(n=1)$. (B) The relative densitometry of Fig. $2 \mathrm{~b}$ ( $n$ $=1)$. (C) The relative densitometry of Fig. $2 \mathrm{C}(n=1)$. (D) The relative densitometry of Fig. $2 \mathrm{~d}(n=1)$. (PPT $186 \mathrm{~kb})$

\section{Abbreviations \\ ADAMTS: A disintegrin-like and metalloproteinase with thrombospondin type 1 motifs; BET: Bromodomain and extraterminal domain; Brd: Bromodomain- containing protein; Cdk9: Cyclin-dependent kinase 9; CycT1: Cyclin T1; DMSO: Dimethyl sulfoxide; GAPDH: Glyceraldehyde-3-phosphate dehydrogenase; H4K12Ac: H4 acetylated at Lys12; H4K5Ac: H4 acetylated at Lys5; H4K8Ac: H4 acetylated at Lys8; IL: Interleukin; JNK: Jun N-terminal kin- ase , MAPK: Mitogen-activated protein kinase; MMP: Matrix metalloproteinase NF-kB: Nuclear factor-kB; OA: Osteoarthritis; $\mathrm{p}$-TEFb: Positive transcription elongation factor b; RNAP II CTD: RNA polymerase II C-terminal domain; S2P Pol II: Ser2-phosphorylated RNA polymerase II C-terminal domain; TNF: Tumor necrosis factor}

\section{Acknowledgements}

We would like to thank Ling Qin for helpful discussions.

\section{Funding}

This study was supported by the National Science Foundation for Distinguished Young Scholars of China (81125013) and National Natural Science Foundation of China (81472116).

\section{Availability of data and materials}

The datasets used and/or analyzed during the current study are available from the corresponding author on reasonable request.

\section{Authors' contributions}

JD, SZ, MZ, XG, HT, and QJ have made substantial contributions to the conception and design and the analysis and interpretation of data and have been involved in the drafting of the manuscript or revising it critically for important intellectual content. QG acquired the data and have been involved in the drafting of the manuscript. JZQ, $J, H J, Y C$, and $C L$ made contributions to the interpretation of data and the drafting of the manuscript. All authors read and approved the final manuscript.

\section{Ethics approval and consent to participate}

Human samples were obtained with informed consent from the donors. The study was approved by the ethical committee of Drum Tower Hospital,

Medical School, Nanjing University.

\section{Consent for publication}

Not applicable.

\section{Competing interests}

The authors declare that they have no competing interests.

\section{Publisher's Note}

Springer Nature remains neutral with regard to jurisdictional claims in published maps and institutional affiliations.

\section{Author details}

${ }^{1}$ The Center of Diagnosis and Treatment for Joint Disease, Drum Tower Hospital, Medical School, Nanjing University, Zhongshan Road 321, Nanjing 210008, Jiangsu, People's Republic of China. ${ }^{2}$ The Model Animal Research Center of Nanjing University, Xuefu Road, Nanjing 210032, Jiangsu, People's Republic of China. ${ }^{3}$ State Key Laboratory of Analytical Chemistry for Life Science, Nanjing University, Hankou Road, Nanjing 210093, People's Republic of China. ${ }^{4}$ Collaborative Innovation Center of Advanced Microstructures, National Laboratory of Solid State Microstructure and Department of Physics, Nanjing University, Hankou Road, Nanjing 210093, People's Republic of China. ${ }^{5}$ Sir Charles Gairdner Hospital, School of Surgery, The University of Western Australia, 35 Stirling Highway, Perth 6009, Australia. ${ }^{6}$ State Key Laboratory of Pharmaceutical Biotechnology and Jiangsu Key Laboratory of Molecular Medicine, Model Animal Research Center and School of Medicine, Nanjing University, Nanjing 210093, People's Republic of China.

Received: 3 July 2018 Accepted: 5 February 2019

Published online: 20 February 2019

\section{References}

1. Kapoor M, Martel-Pelletier J, Lajeunesse D, Pelletier JP, Fahmi H. Role of proinflammatory cytokines in the pathophysiology of osteoarthritis. Nat Rev Rheumatol. 2011;7(1):33-42.

2. Bondeson J, Wainwright S, Hughes C, Caterson B. The regulation of the ADAMTS4 and ADAMTS5 aggrecanases in osteoarthritis: a review. Clin Exp Rheumatol. 2008;26(1):139-45.

3. Vincenti MP, Brinckerhoff CE. Transcriptional regulation of collagenase (MMP-1, MMP-13) genes in arthritis: integration of complex signaling pathways for the recruitment of gene-specific transcription factors. Arthritis Res. 2002:4(3):157-64.

4. Filippakopoulos P, Qi J, Picaud S, Shen Y, Smith WB, Fedorov O, et al. Selective inhibition of BET bromodomains. Nature. 2010;468:1067-73.

5. Dai J, Zhou S, Ge Q, Qin J, Chen D, Xu Z, et al. Bi-directional regulation of cartilage metabolism by inhibiting BET proteins-analysis of the effect of IBET151 on human chondrocytes and murine joints. J Orthop Surg Res. 2018;13(1):118.

6. Belkinal AC, Denis GV. BET domain co-regulators in obesity, inflammation and cancer. Nat Rev Cancer. 2012;12(7):465-77.

7. Wu SY, Chiang CM. The double bromodomain-containing chromatin adaptor Brd4 and transcriptional regulation. J Biol Chem. 2007:282(18):13141-5.

8. Nie $M$, Pang $L$, Inoue $H$, Knox AJ. Transcriptional regulation of cyclooxygenase 2 by bradykinin and interleukin-1 beta in human airway smooth muscle cells: involvement of different promoter elements, transcription factors, and histone h4 acetylation. Mol Cell Biol. 2003;23(24):9233-44.

9. Ito K, Barnes PJ, Adcock IM. Glucocorticoid receptor recruitment of histone deacetylase 2 inhibits interleukin-1 beta-induced histone $\mathrm{H} 4$ acetylation on lysines 8 and 12. Mol Cell Biol. 2000;20(18):6891-903.

10. Chen YJ, Chang LS. NFKB- and AP-1-mediated DNA looping regulates matrix metalloproteinase-9 transcription in TNF-a-treated human leukemia U937 cells. Biochim Biophys Acta. 2015;1849(10):1248-59.

11. Gebauer M, Saas J, Sohler F, Haag J, Söder S, Pieper M, et al. Comparison of the chondrosarcoma cell line SW1353 with primary human adult articular 
chondrocytes with regard to their gene expression profile and reactivity to IL-13. Osteoarthr Cartil. 2005;13:697-708.

12. Tew SR, Murdoch AD, Rauchenberg RP, Hardingham TE. Cellular metnods in cartilage research: primary human chondrocytes in culture and chondrogenesis in human bone marrow stem cells. Methods. 2008:45:2-9.

13. Teng H, Wu B, Zhao K, Yang G, Wu L, Wang R. Oxygen-senesitive mitochondrial accumulation of cystathionine $\beta$-synthase meidiated by Lon protease. Proc Natl Acad Sci U S A. 2013;1 10:12679-84.

14. Yang Z, Yik JH, Chen R, He N, Jang MK, Ozato K, et al. Recruitment of $p$ TEFb for stimulation of transcriptional elongation by the bromodomanin protein Brd4. Mol Cell. 2005;19:535-45.

15. Jang MK, Mochizuki K, Zhou M, Jeong HS, Brady JN, Ozato K. The bromodomain protein Brd4 is a positive regulatory component of $\mathrm{p}$-TEFb and stimulates RNA polymerase II-dependent transcription. Mol Cell. 2005;19:523-34.

16. Sims RJ 3rd, Belotserkovskaya R, Reinberg D. Elongation by RNA polymerase II: the short and long of it. Genes Dev. 2004;18:2437-68.

17. Hargreaves DC, Horng T, Medzhitov R. Control of inducible gene expression by signal-dependent transcriptional elongation. Cell. 2009;138:129-45.

18. Dey A, Chitsaz F, Abbasi A, Misteli T, Ozato K. The double bromodomain protein Brd4 binds to acetylated chromatin during interphase and mitosis. Proc Natl Acad Sci U S A. 2003;100:8758-63.

19. LeRoy G, Rickards B, Flint SJ. The double bromodomain proteins Brd2 and Brd3 couple histone acetylation to transcription. Mol Cell. 2008;30:51-60.

20. Chen WL, Sheu JR, Hsiao CJ, Hsiao SH, Chung CL, Hsiao G. Histone deacetylase inhibitor impairs plasminogen activator inhibitor-1 expression via inhibiting TNF-a-activated MAPK/AP-1 signaling cascade. Biomed Res Int. 2014;2014:231012.

21. Wang $Y$, Wang $Y$, Luo $M$, Wu H, Kong L, Xin $Y$, et al. Novel curcumin analog C66 prevents diabetic nephropathy via JNK pathway with the involvement of p300/CBP-mediated histone acetylation. Biochim Biophys Acta. 2015; 1852(1):34-46.

22. Young DA, Lakey RL, Pennington CJ, Jones D, Kevorkian L, Edwards DR, et al. Histone deacetylase inhibitors modulate metalloproteinase gene expression in chondrocytes and block cartilage resorption. Arthritis Res Ther 2005:R503-12.

23. Culley KL, Hui W, Barter MJ, Davidson RK, Swingler TE, Destrument AP, et al. Class I histone deacetylase inhibition modulates metalloproteinase expression and blocks cytokine-induced cartilage degradation. Arthritis Rheumatol. 2013;65:1822-30

24. Angiolilli C, Kabala PA, Grabiec AM, Van Baarsen IM, Ferguson BS, García S, et al. Histone deacetylase 3 regulates the inflammatory gene expression programme of rheumatoid arthritis fibroblast-like synoviocytes. Ann Rheum Dis. 2017;76(1):277-85.

25. Ziesché E, Kettner-Buhrow D, Weber A, Wittwer T, Jurida L, Soelch J, et al. The coactivator role of histone deacetylase 3 in IL-1-signaling involves deacetylation of p65 NF-kB. Nucleic Acids Res. 2013;41(1):90-109.

Ready to submit your research? Choose BMC and benefit from:

- fast, convenient online submission

- thorough peer review by experienced researchers in your field

- rapid publication on acceptance

- support for research data, including large and complex data types

- gold Open Access which fosters wider collaboration and increased citations

- maximum visibility for your research: over $100 \mathrm{M}$ website views per year

At $\mathrm{BMC}$, research is always in progress.

Learn more biomedcentral.com/submissions 